\title{
LXXXII. Space-time manifolds and corresponding gravitational fields
}

\section{Wilfrid Wilson B.Sc.}

To cite this article: Wilfrid Wilson B.Sc. (1920) LXXXII. Space-time manifolds and corresponding gravitational fields, Philosophical Magazine Series 6, 40:240, 703-712, DOI: 10.1080/14786441208636181

To link to this article: http://dx.doi.org/10.1080/14786441208636181

曲 Published online: 08 Apr 2009.

Submit your article to this journal $\asymp$

Џ Article views: 4

Q View related articles $\square$

Citing articles: 6 View citing articles 5 


\section{Summary.}

1. The hydrodynamic basis of convection of heat suggests study by means of models.

2. We have the formula:

Natural convection . . $h=(k \theta / l) \mathrm{F}\left(c^{2} g l^{3} a \theta / k^{2}\right)$,

Forced convection . , $h=(k \theta / l) \mathbf{F}(l v c / k)$.

3. Gruphs drawn with $h \div(h \theta / l)$ as ordinate and either $\left(c^{2} g / l^{3} a \theta / k^{2}\right)$ or $(l v c / k)$ as abscissa should be independent of the size of the object; consequently, to ascertain the lseat-loss for any particular body it should be necessary only to perform the appropriate experiment with a model.

4. For natural convection Péclet's data have been analysed witl promising result. The formula would not be applicable to bodies where the fluid expansion caused was no longer negligible as a mere volume change.

5 . For forced convection the formula, tested by data given by Hughes, is very promising. 'The cooling fluid is not heated so much as in natural convection, and can still be regarded as incompressible for smaller bodies at higher temperatures. The formula is good, even for thin wires, and it is satisfactory to trace in it the hydrodynamic variable determining turbulence.

6. Evidence available in published data indicates that, for lieat-loss from a body, an excellent first approximation can be obtained from experiments with a model. The principle of similitude affords a convenient method of expressing experimental results.

March 1920.

LXXXII. Space-Time Manifolds and corresponding Gravitational. Fields. By Wilfris Wis.sos, B.Sc., Northampton Polytechic Institute*.

THE main purpose of the present paper is the investigation of the gravitational field of an infinite uniform rectilinear distribution of mass or, more precisely stated, the determination of the equations of the geodesics in a spacetime manifold in which the square of the element of length has the form

$$
d s^{2}=-f_{1} d r^{2}-f_{2} d z^{2}-f_{3} r^{2} d \phi^{2}+f_{4} d t^{2}, \quad . \quad .
$$

where the $f^{\prime \prime}$ s are functions of $r$ only $t$.

* Communicated by $\mathrm{Dr}$. Wm. Wilson.

+ When $f_{1}=f_{2}=f_{3}=f_{1}=1, r z$ and $\phi$ are the ordinary cylindrical space co-ordinates. 
So far as the writer is aware, the only gravitational field which has been investigated from the point of view of Einstein's theory is that of a single particle or of a number of isolated particles. On Newton's theory the intensity of the field in the neighbourhood of such an intinite rectilinear distribution of mass is equal to $\frac{2 m}{r}$, where $m$ is the mass per unit length, using gravitational units. Tue following investigation show's that the intensity as given by the general theory of relativity is, to an exceedingly close approximation, equal to the Newtonian result.

Before proeeding to the actual investigation it will bo well to study the following simpler types of manifold in which the square of the line element has the forms :-

$$
\begin{aligned}
& d s^{2}=-d x^{2}-d y^{2}-d z^{2}-2 \alpha t d x d t+\left(1-\alpha^{2} t^{2}\right) d t^{2}, \\
& d s^{2}=-d r^{2}-d z^{2}-r^{2} d \phi^{2}-2 \omega r^{2} d \phi d t+\left(1-r^{2} \omega^{2}\right) d t^{2}, \\
& d s^{2}=-\mathrm{A} d r^{2}-d z^{2}-r^{2} d \phi^{2}+\mathrm{B} d t^{2}, \quad . \quad . \quad . \quad .
\end{aligned}
$$

where $\alpha, \omega, A$, and $B$ are constants.

In the relativity theory of gravitation the general form of the square of the element of length is

$$
d s^{2}=\sum_{\kappa \tau}^{1,2,3,4} g_{\kappa \tau} d x_{\kappa} d x_{\tau} .
$$

The potentials $g_{k r}$ satisfy the equations

where

$$
\mathrm{G}_{\mu \nu}=0, \text {. . . . . . . }
$$

$$
\begin{aligned}
& G_{\mu \nu} \equiv \sum_{\rho \epsilon}^{1,2,3,4}\left(\Gamma_{\mu \rho}^{\epsilon} \Gamma_{\nu \epsilon}^{\rho}-\frac{\partial}{\partial x_{\rho}} \Gamma_{\mu \nu}^{o}+\frac{\partial^{2}}{\partial u_{\mu} \partial u_{\nu} \nu} \log \sqrt{g} \bar{g}\right. \\
& \left.-\Gamma_{\mu \nu}^{\epsilon} \partial \partial v_{\epsilon} \log \sqrt{g}\right)^{*}
\end{aligned}
$$$$
\Gamma_{\beta \gamma}^{a} \equiv \frac{1}{2} \sum_{\delta}^{1,2,3,4} g^{\mu \delta \delta}\left(\frac{\partial y^{\prime} \beta \hat{i}}{\partial x_{\gamma}}+\frac{\partial y_{\gamma \delta}}{\partial c_{\beta j}}-\frac{\partial y_{\beta \gamma}}{\partial x_{\delta}}\right) .
$$

In some cases the potentials $y_{\kappa \tau}$ may also satisfy the equations

$$
\mathrm{B}_{\mu \nu^{\prime} \sigma}^{\prime \prime}=0, \quad \text {. . . . . . . }
$$

* Einstein, Ann. d. Phys. xlix. p. 769 (1916). Einstein only uses co-ordinates $\mathrm{f}(\mathrm{r}$ which $g=1$. See also Lddington, 'Report on Relative 'Theory of Gravitatios.' 
where

$$
\mathrm{B}_{\mu \nu \sigma}^{\prime \prime} \equiv \sum_{\epsilon}^{1,2,3,4}\left(\Gamma_{\mu \sigma}^{\epsilon} \Gamma_{\epsilon \nu}^{\rho}-\Gamma_{\mu \nu}^{\epsilon} \Gamma_{\epsilon \sigma}^{\rho}+\frac{\partial}{\partial v_{\nu}} \Gamma_{\mu \sigma}^{\rho}-\frac{\partial}{\partial x_{\sigma}} \Gamma_{\mu \nu}^{\rho}\right) .
$$

The latter equation expresses the necessary and sufficient condition that, by a suitable choice of coordinates, the square of the element of length can be put in the form :-

$$
d s^{2}=\sum_{s}^{1,2,3,4} d x_{s}^{2} \quad . \quad . \quad . \quad . \quad .
$$

and the gravitational field made to vanish everywhere. Such a field may conveniently be termed a non-permanent one. The manitolds (2), (3), and (4) furnish simple illustrations of such fields.

In the manifold (2) the values of the $g_{\kappa \tau}$ and $g^{\kappa \tau}$ are :-

$$
\left\{\begin{array} { l l } 
{ g _ { 1 1 } = - 1 } \\
{ y _ { 2 2 } = - 1 } \\
{ g _ { 3 3 } = - 1 } \\
{ g _ { 4 4 } = 1 - \alpha ^ { 2 } t ^ { 2 } } \\
{ g _ { 1 4 } = - \alpha t }
\end{array} \quad \left\{\begin{array}{l}
g^{11}=\alpha^{2} t^{2}-1 \\
g^{22}=-1 \\
g^{33}=-1 \\
g^{44}=1 \\
g^{14}=-\alpha t
\end{array}\right.\right.
$$

and

$$
g=-1
$$

where $g$ is the determinant of the $g_{\kappa r}$.

The Christoffel expressions $\Gamma_{\beta \gamma}^{a}$ all vanish with the exception of $\Gamma_{44}^{4}$, which has the value

The equations

$$
\Gamma_{44}^{\prime}=\alpha \text {. }
$$

$$
\frac{\partial^{2} x_{\sigma}}{\partial s^{2}}+\sum_{\alpha \beta}^{1,2,3,4} \Gamma_{\alpha \beta}^{\sigma} \frac{\partial x_{\alpha}}{\partial s} \frac{\partial x_{\beta}}{\partial s}=0, \quad \sigma=1,2,3,4, .
$$

of the geodesics then give the following equations of motion :-

$$
\begin{aligned}
& \frac{d^{2} x}{d s^{2}}=-a c^{2}, \\
& \frac{d^{2} y}{d s^{2}}=0 \\
& \frac{d^{2} z}{d s^{2}}=0 \\
& d s^{2}=0
\end{aligned}
$$


$70 b$ Mr. W. Wilson on Space. Time Manifolds

where the constant $c$ is given by

$$
\frac{d t}{d s}=c .
$$

The first of these equations may now be written

$$
\frac{d^{2} x}{d t^{2}}=-\alpha
$$

showing that the manifold (2) corresponds to a uniform gravitational field in the $x$ direction.

In the manifold (3) we have

$$
\left\{\begin{array}{l}
g_{11}=-1 \\
g_{22}=-1 \\
g_{33}=-r^{2} \\
g_{14}=1-r^{2} \omega^{2} \\
g_{34}=-r^{2} \omega
\end{array}\right.
$$$$
\left\{\begin{array}{l}
g^{11}=-1 \\
g^{22}=-1 \\
g^{33}=\omega^{2}-\frac{1}{r^{2}} \\
g^{44}=1 \\
g^{34}=-\omega,
\end{array}\right.
$$

and

$$
g=-r^{2} .
$$

The non-vanishing Cluristoffel expressions $\Gamma_{\beta \gamma}^{\alpha}$ are:

$$
\begin{array}{ll}
\Gamma_{33}^{\prime}=-r, & \Gamma^{3}{ }_{13}=\frac{1}{r}, \\
\Gamma_{34}^{\prime}=-\omega r, & \Gamma^{3}{ }_{14}=\frac{\omega}{r}, \\
\Gamma_{41}^{\prime}=-\omega^{2} r ; &
\end{array}
$$

and the equations of motion (8) are :

$$
\begin{aligned}
\frac{d^{2} r}{d s^{2}}-r\left\{\frac{d(\phi+\omega t)}{d s}\right\}^{2} & =0, \\
\frac{d^{2} \phi}{d s^{2}}+\frac{2}{r} \frac{d r}{d s} \frac{d(\phi+\omega t)}{d s} & =0, \\
d^{2}= & =0, \\
d s^{2} & =0 . \\
\frac{d^{2} t}{d s^{2}} & =0
\end{aligned}
$$

The last equation gives

$$
{ }_{d s}^{d t}=c,
$$


where $c$ is a constant. Substituting this value of $\frac{d t}{d s}$ in the first equation, we obtain

$$
\frac{d^{2} r}{d t^{2}}-r\left(\frac{d \phi}{d t}+\omega\right)^{2}=0
$$

and taking $\omega$ to be an angular velocity we have the equations of motion in a centrifugal field.

In the manifold (4) the non-vanishing Christoffel expressions are

$$
\Gamma_{13}^{3}=\frac{1}{r} \quad \text { and } \quad \Gamma_{33}^{\prime}=-\frac{r}{\mathrm{~A}} .
$$

The equations of motion ( 8 ) become

$$
\left.\begin{array}{rl}
\frac{d^{2} r}{d s^{2}}-\frac{r}{\mathrm{~A}}\left(\frac{d \phi}{d s}\right)^{2} & =0, \\
\frac{d^{2} \phi}{d s^{2}}+\frac{2}{r} \frac{d r}{d s} \frac{d \phi}{d s} & =0, \\
\frac{d^{2} z}{d s^{2}} & =0, \\
\frac{d^{2} t}{d s^{2}} & =0 .
\end{array}\right\}
$$

If, instead of $\phi$ we use $\phi^{\prime}$, where

$$
\phi=\sqrt{\bar{A} \phi^{\prime}},
$$

the equations (9) become

$$
\begin{aligned}
\frac{d^{2} r}{d s^{2}}-r\left(\frac{d \phi^{\prime}}{d s}\right)^{2} & =0 \\
\frac{d^{2} \phi^{\prime}}{d s^{2}}+\frac{2}{r} \frac{d r}{d s} \frac{d \phi^{\prime}}{d s} & =0 \\
\frac{d^{2} z}{d s^{2}} & =0, \\
d^{2} t & =0 . \\
d s^{2} & =0
\end{aligned}
$$

We may therefore regard equations (9) as the equations of motion of a particle moving with uniform velocity in a straight line with respect to an inertia system $\left(r, z, \dot{\phi}^{\prime}\right)$, 
viewed from a system $(r, z, \phi)$ rotating in the inertia system with angular velocity $(1-\sqrt{A})$ times that of the particle in the inertia systcm.

We now proceed to the investigation of the manifold (1), where

$$
d s^{2}=-\dot{f}_{1} d r^{2}-f_{2} d z^{2}-f_{3} r^{2} d \phi^{2}+\dot{f_{4}} d t^{2},
$$

and the functions

$$
-f_{1}(r), \quad-f_{2}(r), \quad-r^{2} f_{3}(r), \quad f_{4}^{\prime}(r)
$$

must be solutions of the equations (5).

For our purpose there is no loss in generality in using any function of $r$ in place of $r$. We therefore write

$$
-r^{2} f_{3}^{\prime}(r)=-r^{\prime 2} \quad \cdot . \cdot .
$$

and obtain for the square of the line element the form :

$$
d s^{2}=-f_{1}^{\prime} d r^{2}-f_{2}^{\prime} d z^{2}-r^{2} d \phi^{2}+f_{4} d t^{2}, \quad . \quad .
$$

where the accent has been dropped after making the substitution (10).

It is easier to deal with the equations (5) if we write $d s^{2}$ in the form

$$
d s^{2}=-e^{\lambda} d r^{2}-e^{\mu} d z^{2}-r^{2} d \phi^{2}+e^{\nu} d t^{2}, \quad . \quad(12) \dagger
$$

where $e^{\lambda}=f_{1}^{\prime}\left(r^{\circ}\right)$ etc., and $\lambda, \mu$, and $v$ are functions of $r$ which have to be determined.

The Christoffel expressions $\Gamma_{\beta}^{\alpha}$ are then found to be

$$
\left\{\begin{array} { l } 
{ \Gamma _ { 1 1 } ^ { \prime } = \frac { 1 } { 2 } \lambda ^ { \prime } , } \\
{ \Gamma _ { 2 2 } ^ { \prime } = - \frac { 1 } { 2 } \mu ^ { \prime } e ^ { \mu - \lambda } , } \\
{ \Gamma _ { 3 3 } ^ { \prime } = - r e ^ { - \lambda } , } \\
{ \Gamma _ { 4 4 } ^ { \prime } = \frac { 1 } { 2 } \nu ^ { \prime } e ^ { \nu - \lambda } , }
\end{array} \quad \left\{\begin{array}{l}
\Gamma_{12}^{2}=\frac{1}{2} \mu^{\prime}, \\
\Gamma_{13}^{3}=\frac{1}{\gamma} \\
\Gamma_{14}^{4}=\frac{1}{2} \nu^{\prime}
\end{array}\right.\right.
$$

where

$$
\lambda^{\prime}=\frac{\partial \lambda}{\partial r^{\prime}}, \quad \mu^{\prime}=\frac{\partial \mu}{\partial r}, \quad \nu^{\prime}=\frac{\partial \nu}{\partial r} .
$$

Substituting these expressions in the equations (5). the

* I am indebted to Dr. Wilson, of King's College, for suggesting the investigation of a line element of this type.

+ E'dington, Report. 
latter become :

$$
\begin{aligned}
& \frac{1}{2} \mu^{\prime \prime}-\frac{1}{4} \lambda^{\prime} \mu^{\prime}+\frac{1}{4} \mu^{\prime 2}=-\frac{1}{2} \nu^{\prime \prime}+\frac{1}{2} \frac{\lambda^{\prime}}{r}+\frac{1}{4} \lambda^{\prime} \nu^{\prime}-\frac{1}{4} \nu^{\prime 2}, . \\
& \frac{1}{2} \mu^{\prime \prime}-\frac{1}{4} \lambda \mu^{\prime}+\frac{1}{4} \mu^{\prime 2}=-\frac{1}{2}{ }_{r}^{\prime}-\frac{1}{4} \mu^{\prime} \nu^{\prime}, \text {. . . . . } \\
& \lambda^{\prime}=\mu^{\prime}+\nu^{\prime}, \quad . \quad . \quad . \quad . \quad . \quad . \\
& \frac{1}{2} \frac{\nu^{\prime}}{\gamma^{\prime}}+\frac{1}{4} \mu^{\prime} \nu^{\prime}=-\frac{1}{2} \nu^{\prime \prime}+\frac{1}{4} \lambda^{\prime} \nu^{\prime}-\frac{1}{4} \nu^{\prime 2} . . .
\end{aligned}
$$

Substituting the value of $\lambda^{\prime}$ given by (15) in (13), (14), and (16), we obtain the equations :

$$
\begin{aligned}
& \mu^{\prime \prime}+\nu^{\prime \prime}-\mu^{\prime} \nu^{\prime}=\frac{\mu^{\prime}}{r}+\frac{\nu^{\prime}}{r}, \quad . \quad . \quad . \quad . \\
& \mu^{\prime \prime}+\frac{\mu^{\prime}}{r}=0, \quad . \quad . \quad . \quad . \quad . \\
& \nu^{\prime \prime}+\frac{\nu^{\prime}}{r}=0 . \quad . \quad . \quad . \quad . \quad .
\end{aligned}
$$

From (18) and (19) we find

$$
\begin{aligned}
\mu^{\prime} & =-\frac{4 m}{r}, \\
\nu^{\prime} & =\frac{a}{r^{2}} ;
\end{aligned}
$$

and therefore from (15)

$$
\lambda^{\prime}=\frac{-4 m+a}{r}
$$

where $4 m$ and $a$ are constants of integration.

Substituting these values of $\mu^{\prime}$ and $\nu^{\prime}$ in (17) we see that

whence

$$
a=\frac{4 m}{1-2 m} \text {; }
$$

and thetefore

$$
-4 m+a=\frac{8 m^{2}}{1-2 m}
$$

$$
\begin{aligned}
\mu^{\prime} & =-\frac{4 m}{r^{\prime}}, \\
\nu^{\prime} & =\frac{4 m}{1-2 m} \cdot \frac{1}{r^{\prime}} \\
\lambda^{\prime} & =\frac{8 m^{2}}{1-2 m} \cdot \frac{1}{r^{\circ}}
\end{aligned}
$$


Integrating these last equations, we obtain

$$
\left.\begin{array}{l}
\mu=-4 m \log r+\mathrm{A} \\
\nu=\frac{4 m}{1-2 m} \log r+\mathrm{B} \\
\lambda=\frac{8 m^{2}}{1-2 m} \log r+\mathrm{C},
\end{array}\right\} . . .
$$

where A, B, and C are constants of integration.

We shall see, when the equations of motion are written down, that $m$ can be identified with the mass per unit length of the $z$ axis. When $m$ is zero the square of the element of length will take the form

$$
d s^{2}=-d r^{2}-d z^{2}-r^{2} d \phi^{2}+d t^{2} ;
$$

i.e., when $m=0$,

Therefore

$$
\lambda=0, \quad \mu=0, \quad \nu=0 .
$$

$$
\mathrm{A}=0, \quad \mathrm{~B}=0, \quad(\mathrm{Y}=0,
$$

and the values of $\lambda, \mu$, and $\nu$ are

$$
\begin{aligned}
\mu & =-4 m \log r \\
\nu & =\frac{4 m}{1-2 m} \log r \\
\lambda & =\frac{8 n^{2}}{1-2 m} \log r
\end{aligned}
$$

The square of the element of length (1) is therefore

$$
d s^{2}=-r^{\left(\frac{8 m^{2}}{1-2 m}\right)} d r^{2}-r^{-4 m} d z^{2}-r^{2} d \phi^{2}+r^{\left(\frac{4 m}{1-2 m}\right)} d t^{2} .
$$

The Christoffel expressions which do not vanish are :-

$$
\begin{array}{ll}
\Gamma_{11}^{\prime}=\frac{4 m^{2}}{1-2 m} \cdot \frac{1}{r}, & \Gamma_{12}^{\prime \prime}=\frac{-2 m}{r}, \\
\Gamma_{22}^{\prime}=\frac{2 m}{r} \cdot r^{\left(\frac{-4 m}{1-2 m}\right)}, & \Gamma_{13}^{3}=\frac{1}{r} \\
\Gamma_{33}^{\prime}=-r^{\left(1-\frac{8 m^{2}}{1-2 m}\right)}, & \Gamma^{4}{ }_{14}=\frac{2 m}{1-2 m} \cdot \frac{1}{r} . \\
\Gamma_{44}^{\prime}=\frac{2 m}{1-2 m} \cdot r^{(4 m-1)},
\end{array}
$$


Substituting these values in the equations (8), we obtain for the geodesics the equations following :-

$$
\begin{aligned}
\frac{d^{2} r}{d s^{2}}+\left(\frac{4 m^{2}}{1-2 m}\right) \frac{1}{r}\left(\frac{d r}{d s}\right)^{2}+2 m r^{\left(-1-\frac{4 m}{1-2 m}\right)}\left(\frac{d z}{d s}\right)^{2} & \\
-r^{\left(1-\frac{8 m^{2}}{1-2 m}\right)}\left(\frac{d \phi}{d s}\right)^{2}+\frac{2 m}{1-2 m} r^{(4 m-1)}\left(\frac{d t}{d s}\right)^{2} & =0, \\
\frac{d^{2} \phi}{d s^{2}}+\frac{2}{r} \frac{d r}{d s} \frac{d \phi}{d s} & =0, \\
\frac{d^{2} z}{d s^{2}}-\frac{4 m}{r} \frac{d r}{d s} \frac{d z}{d s} & =0, \\
d^{2} t+\left(\frac{4 m}{1-2 m}\right) \frac{1}{r} \frac{d r}{d s} \overline{d t} & =0 . \\
d s^{2}+\left(\frac{1-2}{d s}\right. &
\end{aligned}
$$

We may interpret these as the equations of motion of a particle in the gravitational field of an infinitely extended uniform rectilinear distribution of mass along the $z$ axis. From (25) we see that if the particle is moving initially in the plane $z=$ constant, it must remain in this plane: i.e., we have always

$$
\frac{d z}{d s}=0 . \quad . \quad . \quad . \quad . \quad .
$$

From (24) we have

$$
r^{2} \frac{d \phi}{d s}=h
$$

where $h$ is a constant of integration. This equation simply states that a radius vector sweep's out equal areas in equal times. From (26) we get

$$
\frac{d t}{d s}=c r^{\left(\frac{-4 m}{1-2 m}\right)}
$$

where $c$ is a constant of integration.

Substituting these values of $\frac{d z}{d s}$ and $\frac{d t}{d s}$ from $\left(25^{\prime}\right)$ and $\left(26^{\prime}\right)$ in (23), we write (23) and (24) in the form :

$$
\begin{aligned}
\frac{d^{2} r}{d s^{2}}-r^{\left(1-\frac{8 m^{9}}{1-2 m}\right)}\left(\frac{d \phi}{d s}\right)^{2} & =\left\{\frac{-2 m}{r} \frac{c^{2}}{1-2 m} r^{\left(\frac{-8 m^{2}-4 m}{1-2 m}\right)}\right. \\
& \left.-\frac{4 m^{2}}{r(1-2 m)}\left(\frac{d r}{d s}\right)^{2}\right\}, \\
r^{2} \frac{d \phi}{d s} & =\text { h. . . . . . . . . . . }
\end{aligned}
$$




\section{Space-Time Manifolds and Graritutional Fields.}

The Newtonian equations of motion for the type of field we are dealing with are :

$$
\begin{aligned}
& \frac{d^{2} r^{2}}{d t^{2}}-r\left(\frac{d \phi}{d t}\right)^{2}=-\frac{2 m}{r} . . \quad . \quad . \\
& r^{2} \frac{d \phi}{d t}=h . \quad \text {. . . . . . }
\end{aligned}
$$

Comparing these with (27) and (28) we notice they are identical for a sufficiently small $m$. Since we are using gravitational units, $m$ is, for any solid cylinder of laboratory dimensions, negligible compared willi unity and we see that (27) takes the form

$$
\frac{d^{2} r}{d s^{2}}-r\left(\frac{d \phi}{d s}\right)^{2}=-\frac{2 m}{r} c^{2} . \quad . \quad . \quad .
$$

But for small $m,\left(26^{\prime}\right)$ gives

$$
\frac{d t}{d s}=c
$$

and substituting $\frac{d t}{d s}$ for $c$ in (29) we obtain the Newtonian equation $\left(27^{\prime}\right)$. When $m$, however, is rery great the equations of motion are more complicated. It is instructive to put equation (27) in the approximate form obtained by neglecting small quantities of the second order. On eliminating $d s$ by means of equation $\left(26^{\prime}\right)$ we obtain

$$
\frac{d^{2} r}{d t^{2}}-r\left(\frac{d \phi}{d t}\right)^{2}=-\frac{2 m}{r^{1-4 m}}
$$

neglecting quantities of the order of $m^{2}$ and assuming the radial velocity component $\frac{d r^{2}}{d t}$ to he small or zero. To this order of approximation therefore, and with the assumption just mentioned, we may take $-\frac{1}{2}, g_{44}$ to be the gravitational potential of the field we have been investigating, since

$$
\frac{\partial}{\partial r}\left(-\frac{1}{2} g_{44}\right)=-\frac{1}{2} \frac{\partial}{\partial r}\left(r^{\frac{4 m}{2 m}}\right)
$$

gives for the intensity of the field the expression obtained above.

Northampton Institute, E.C. 1. 20 th July, 1920. 